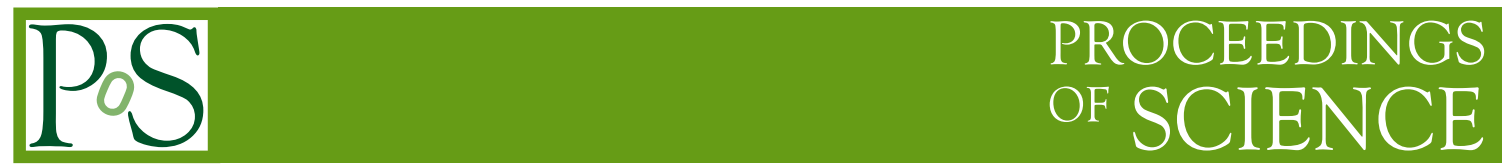

\title{
Theory developments in differential top-quark distributions
}

\section{David Heymes*}

Cavendish Laboratory, University of Cambridge, Cambridge CB3 OHE, UK

E-mail: dheymes@hep.phy.cam.ac.uk

Differential distributions for on-shell top-quark pair production at next-to-next-to-leading order in perturbative QCD for the Large Hadron Collider at $\sqrt{s}=8 \mathrm{TeV}$ are presented and discussed. The theoretical framework of the calculation is summarized. By comparing the prediction to measurements, it turns out that next-to-next-to-leading order corrections are necessary in order to resolve a discrepancy between data and next-to-leading order predictions in the transverse momentum distribution of the top-quark.

8th International Workshop on Top Quark Physics

14-18 September, 2015

Ischia, Italy

${ }^{*}$ Speaker. 


\section{Introduction}

Top-quarks provide an excellent testing ground for the Standard Model and in particular Quantum Chromodynamics (QCD) at the Large Hadron Collider (LHC). The large number of top-quark pairs that have been produced at $\sqrt{s}=7 \mathrm{TeV}$ and $\sqrt{s}=8 \mathrm{TeV}$ allows for detailed studies of topquark properties in different kinematical regions. Additionally, they are an important background for the main physics program measurements at the LHC at $\sqrt{s}=13 \mathrm{TeV}$, namely precision measurements of the Higgs-boson properties and searches for physics beyond the Standard Model. Precision predictions at a differential level for top-quark pairs are demanded in order to give a reliable interpretation of the measurements within and beyond the Standard Model.

In order to test the Standard Model down to the precision that can be achieved at the LHC, theoretical predictions at the same accuracy need to be provided. The total inclusive cross-section at next-to-next-to-leading order QCD [1, 2, 3, 4, 5, 6] shows a very good agreement with measurements at 7,8 and $13 \mathrm{TeV}[7,8,9,10,11]$. Furthermore, only at this order of perturbation theory the theoretical uncertainty is at the same level as the precision of the measurement.

At a differential level, most measurements of the decay products of the top-quark, namely leptons and jets, are currently in good agreement with next-to-leading order predictions obtained using available Monte Carlo event generators. In contrast, the $p_{\mathrm{T}}$-distribution of the top-quark itself reveals a discrepancy between measurements and next-to-leading order predictions at $\sqrt{s}=7,8$ and $13 \mathrm{TeV}[12,13,14,15,16,17,18]$. Since the top-quark is not measured directly, but reconstructed from its decay products using Monte Carlo generators, inaccuracies in the Standard Model description of the top-quark level process could cause a tensions. Higher-order QCD corrections will help to identify the origin of those discrepancies.

Several approximations of the next-to-next-to-leading order QCD corrections to differential topquark pair production have been presented [19, 20, 21, 22, 23, 24]. These approximations are next-to-leading order accurate and, in addition, they capture contributions of the next-to-next-toleading order result in specific kinematical regions, e.g. the partonic threshold of the top-quark pair.

In this write-up, full next-to-next-to-leading order differential distributions for top-quark pair production at the LHC at $8 \mathrm{TeV}$ are discussed. First, an overview of next-to-next-to-leading order computations for top-quark pair production is presented. This is followed by results for the $p_{\mathrm{T}^{-}}$, $m_{t \bar{t}^{-}}, y_{t \bar{t}^{-}}$and $y_{t}$-distribution. Finally, the results are summarized and an outlook for further studies is given. For additional details please refer to the main publication [25].

\section{Next-to-next-to-leading order computations for top-quark pairs}

In order to perform numerical computations beyond leading order, a subtraction framework is needed to consistently cancel soft and collinear singularities between virtual and real contributions to the cross section. At next-to-leading order general algorithms are established and allow an automated simulation of a large class of processes, that are relevant for the phenomenology at the LHC $[26,27]$. At next-to-next-to-leading order different subtraction schemes have been proposed and applied to single processes.

In the context of top-quark pair production three approaches can be distinguished: The antenna- 
subtraction scheme has been used to calculate differential distributions in the leading colour approximation for the partonic contribution $q \bar{q} \rightarrow t \bar{t}[28,29]$. The $q_{\mathrm{T}}$-subtraction scheme has been applied to top-quark pair production in [30] and results for the $q q^{\prime}$-channel to the total inclusive cross section have been obtained.

The sector improved residue subtraction scheme, STRIPPER, has been proposed in [31] and subsequently successfully applied to the calculation of the total inclusive cross section for top-quark pair production $[1,2,3,4,5,6,32]$. The result includes all partonic processes without further approximations except the truncation of the perturbation series beyond next-to-next-to-leading order. Afterwards, the same framework has been used to predict the forward-backward asymmetry at the Tevatron [33], which has been the first differential prediction for on-shell top-quark pair production at next-to-next-to-leading order.

However, the first formulation of the subtraction scheme and its implementation for the Tevatron setup were not suited to provide robust differential results for the LHC on an adequate time-scale. A substantial diminution of the convergence of the Monte Carlo integration has been observed as the collider energy has been increased. This behaviour is due to large logarithms of ratios between the top-quark mass and the partonic center-of-mass energy in phase space integrals. Moreover, current and future demands of the LHC require a fast Monte Carlo generator, that provides reliable predictions at next-to-next-to-leading order, which can be flexibly adjusted to experimental setups. In view of those requirements, a complete new implementation of STRIPPER has been developed, which is based on an improved four-dimensional formulation of the subtraction scheme [34]. The whole framework is general, since it exploits the process independent soft and collinear factorization of tree-level and one-loop matrix elements as well as the universal singular structure of virtual one-loop and two-loop amplitudes [32, 35, 36, 37, 38, 39, 40, 41, 42, 43, 44, 45, 46, 47, 48]. The remaining information about a specific process under consideration can be provided by interfacing the corresponding tree-level matrix elements as well as the finite parts of the one-loop and two-loop amplitudes. The implemented event generator includes concepts that have been proven and tested at next-to-leading order to increase speed and efficiency of the numerical calculation, e.g. Monte Carlo summation over partonic subprocesses and Monte Carlo summation over external polarizations. The software allows for a simultaneous calculation of different parton distribution functions, different renormalization and factorization scales and different observables.

For the specific case of top-quark pair production the tree-level matrix elements are obtained from Ref. [49]. The four-point one-loop amplitudes have been recomputed independently, but can be found in Refs. [50, 51, 52]. The five-point one-loop amplitudes are taken from the code used in Refs. [53, 54]. The two-loop amplitudes are numerically given in form of a dense grid $[55,56]$. Partial analytic results for the two-loop amplitudes can be found in Refs. [57, 58, 59, 60].

\section{Results}

The differential results presented in Fig. 1 and Fig. 2 are calculated using the following setup. The pole mass of the top-quark is set to $m_{t}=173.3 \mathrm{GeV}$. The distributions have been calculated using the MSTW2008 parton distribution function (PDF) set [61], where PDF uncertainties are not displayed. Each order includes the PDF set of the corresponding order. The theoretical un- 

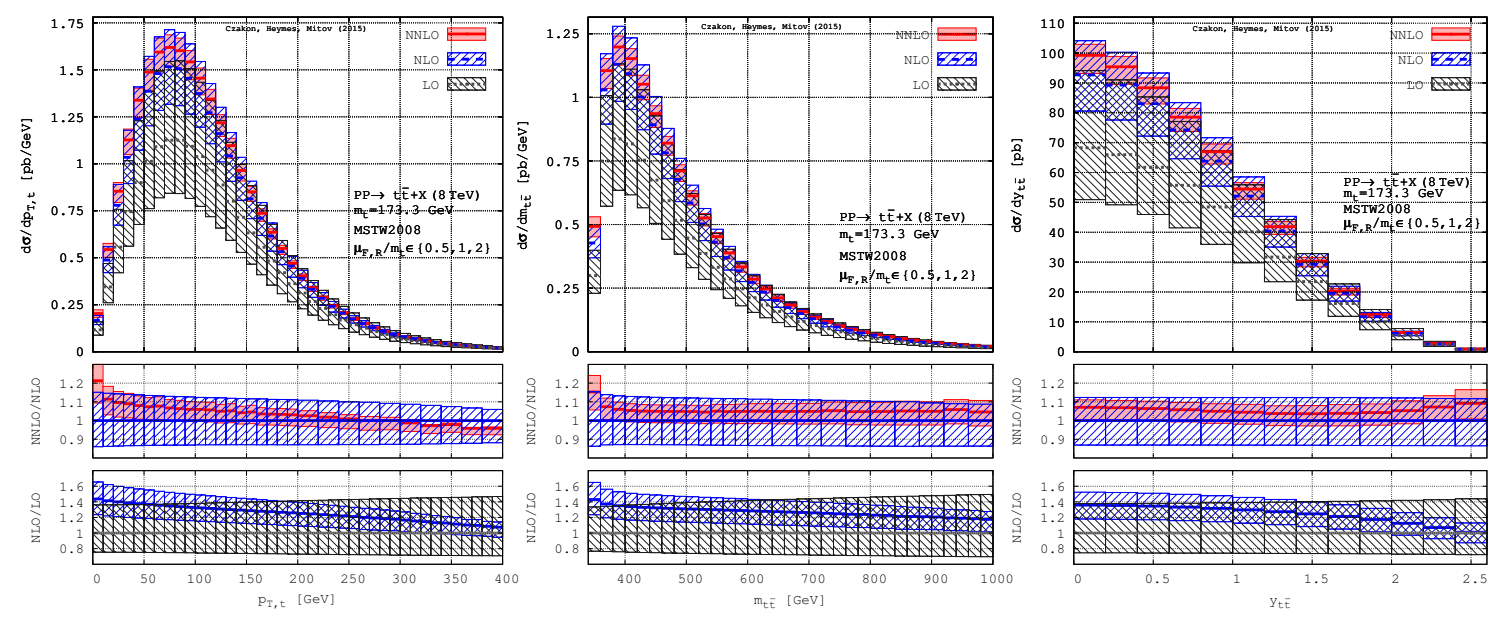

Figure 1: Top/antitop $p_{\mathrm{T}}$-distribution (left), $m_{t \bar{t}}$-distribution (center) and $y_{t t}$-distribution (right) in leading order (LO), next-to-leading order (NLO) and next-to-next-to-leading order (NNLO) QCD. Error bands are from scale variation [25].

certainties are obtained by varying the renormalization scale $\mu_{\mathrm{R}}$ and factorization scale $\mu_{\mathrm{F}}$ independently around the central scale $\mu_{\mathrm{R}}=\mu_{\mathrm{F}}=m_{t}$. The additional restriction, $0.5<\mu_{\mathrm{R}} / \mu_{\mathrm{F}}<2$, is imposed [62]. All results have been cross checked with available results: Integrals over the distributions reproduce the total cross section of Refs. [1, 2, 3, 4] to better than permil level. The nextto-leading order results are cross checked with the Monte Carlo event generator MCFM [63, 64].

Figure 1 shows the $p_{\mathrm{T}, t}, m_{t \bar{t}}$ and $y_{t \bar{t}}$ differential distributions for the absolute normalization as well as the corresponding next-to-leading order and next-to-next-to-leading order K-factors. It should be noticed that the widths of bins of the calculation is much smaller than the widths of the bins that are currently used by the ATLAS and CMS collaborations, which allows to use these results for a variety of phenomenological analyses.

Each of the three distributions shows a good perturbative convergence. This behaviour, which has already been known for the total inclusive cross section, can be observed for each histogram bin separately. The central value lies within the error band of the previous order. This suggests that the theoretical predictions are robust within the stated error bands, while keeping in mind that the uncertainty of the PDF set is not included yet.

A significant rise of the $\mathrm{K}$-factors in the first bin of the $p_{\mathrm{T}, t}$-distribution and to a lesser degree in the first bin of the $m_{t \bar{t}}$-distribution can be observed. However, this enhancement at low $p_{\mathrm{T}}$ and low $m_{t \bar{t}}$ is due to soft-gluon and Coulomb effects at the top-quark pair threshold, which are not captured within a fixed order calculation. Related work, in order to capture these effects to all orders can be found in Refs. [19, 20, 21, 22, 65, 66, 67, 68, 69]. A further investigation of these effects and matching resummed results to the fixed order prediction would be interesting.

The next-to-next-to-leading order K-factor shows a significant slope in the $p_{\mathrm{T}}$-distribution, which, however, is within the next-to-leading error band. The $m_{t \bar{t}}$-distribution at next-to-next-to-leading order is remarkably stable with respect to the next-to-leading order prediction over the whole range of displayed values. It is therefore a well suited observable to look for new resonances beyond the Standard Model as suggested for example in Ref. [70]. The shape of the rapidity distribution of 
the top-quark pair at next-to-next-to-leading order changes little with respect to the next-to-leading order distribution. This can be seen by looking at the next-to-next-to-leading order K-factor. In contrast, the next-to-leading order K-factor shows a rather large change of the shape going from leading order to next-to-leading order. This allows the conclusion that this distribution stabilizes by including next-to-next-to-leading order effects.

A first comparison of the $p_{\mathrm{T}}$-distribution and the $y_{t}$-distribution of the top-quark with the CMS measurement in the lepton and jets channels from Ref. [15] is shown in Fig. 2. Further comparisons with CMS data can be found in Ref. [71], while a comparison with the ATLAS measurements at $\sqrt{s}=8 \mathrm{TeV}$ has been included in Ref. [72].

The distributions are normalized, such that integrating the displayed bins yields unity. Uncertainties due to scale variations for next-to-next-to-leading order distributions are shown exclusively. The lower panel displays the ratio of the Data, the leading order and the next-to-leading order distribution with respect to the next-to-next-to-leading order distribution. There is a slight mismatch between the normalization of the data and the prediction. The transverse momentum distribution of the data includes an additional bin, $400 \mathrm{GeV}<p_{\mathrm{T}}<500 \mathrm{GeV}$, which contributes 4 permil to the normalization. In the rapidity distribution, the last bin of the theory prediction extends up to $\left|y_{t}\right|<2.6$, while the last bin of the measurement extends only up to $\left|y_{t}\right|<2.5$. This can be seen explicitly in Fig. 2.

The first observation is that the tension between data and the next-to-leading order result for the $p_{\mathrm{T}}$-distribution is resolved at next-to-next-to-leading order. In each bin the Standard Model prediction gets closer to the CMS data and an agreement within the displayed uncertainties can be observed. The normalized rapidity distribution of the top-quark is stable with respect to next-tonext-to-leading order corrections. Within the experimental uncertainty the data seems to be well described by the next-to-next-to-leading order prediction.

\section{Summary and outlook}

In this write-up, differential distributions for top-quark pair production at next-to-next-toleading order QCD for the LHC at $\sqrt{s}=8 \mathrm{TeV}$ have been presented. These results have been obtained using a complete independent implementation of the subtraction scheme STRIPPER and include all partonic channels, where no approximations have been made.

These results can be used for further phenomenological studies and tuning of Monte Carlo event generators in order to validate the estimated Standard Model background at the LHC. Moreover, they are valuable to constrain parton distribution functions using LHC data and to measure the strong coupling $\alpha_{s}$. High precision Standard Model prediction allow to further explore limitations of the Standard Model and allow to constrain different scenarios beyond the Standard Model.

The presented results have been obtained for fixed scale settings $\mu_{\mathrm{F}, \mathrm{R}} \in\left\{m_{t}, m_{t} / 2,2 m_{t}\right\}$, which allowed for several cross checks of the final result with previous calculations, e.g. the total inclusive cross section. However, for differential predictions this setting may not be the most appropriate one. In the near future results will become available using several dynamical scales and will include different PDF sets and PDF error estimates.

The calculation will be extended to a center-of-mass energy of $\sqrt{s}=13 \mathrm{TeV}$. In this context, it 

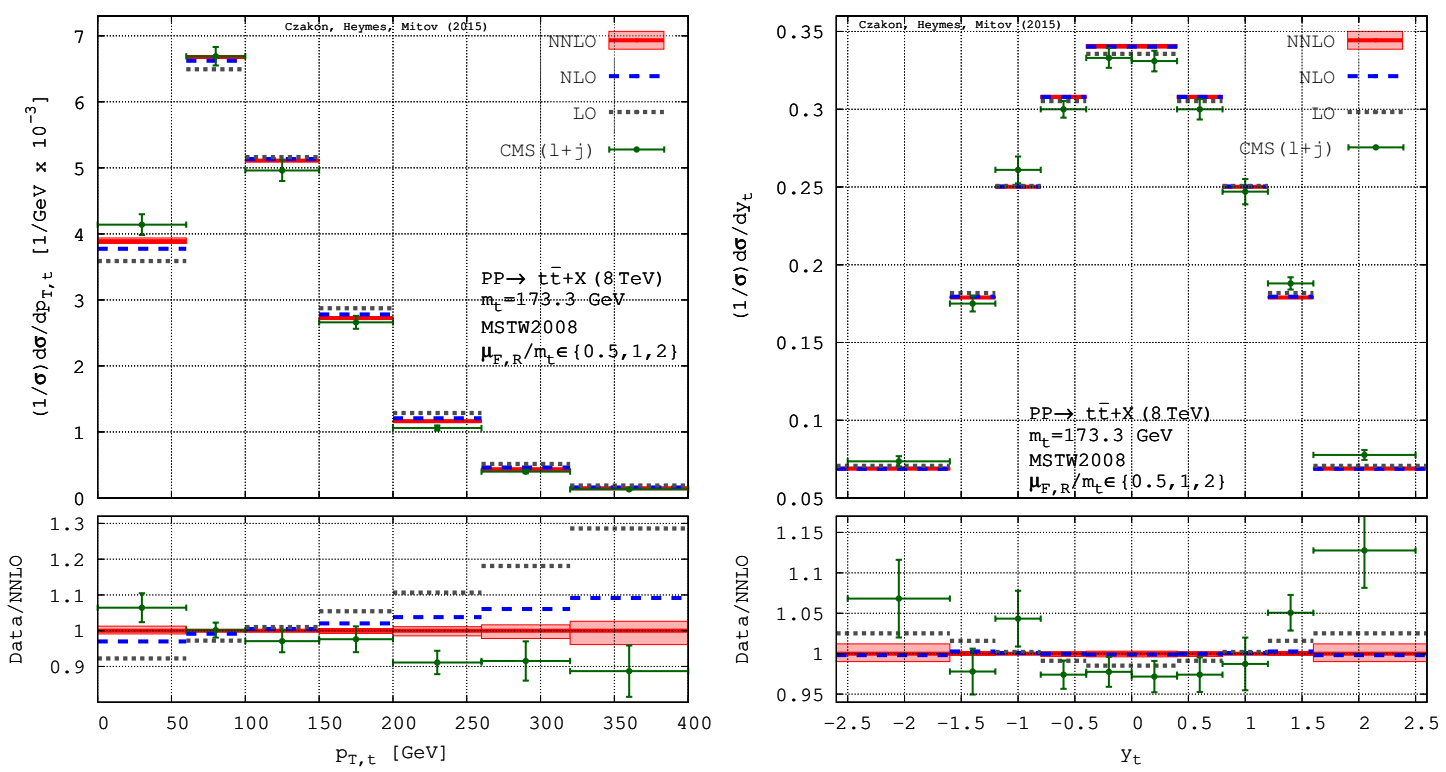

Figure 2: Normalised distribution for the $p_{\mathrm{T}}$ of the top/antitop-quark (left) and the rapdity $y$ of the top/antitop-quark (right) in comparison with CMS measurements [15, 25]

would be interesting to extend the kinematic regime of the $m_{t t^{-}}$and $p_{\mathrm{T}^{-}}$distribution to higher values, since a significant number of top-quark pairs are produced in this so called boosted regime, $\sqrt{\hat{s}} \gg m_{t}$. In this regime large logarithms can be resummed to all orders to improve a fixed order calculation $[73,74]$. A first comparison of the resummed results matched to next-to-leading order QCD and the full next-to-next-to-leading order at result $\sqrt{s}=8 \mathrm{TeV}$ has been presented in Ref. [75]. An investigation of how the resummed result could be matched to the full next-to-nextto-leading order prediction would be interesting.

Finally, a particle level comparison with data would be possible, once decays of the top-quark are included into the calculation at next-to-next-to-leading order. It should be noticed that nextto-next-to-leading order corrections for on-shell top-quark decays have already been presented in Refs. [76, 77].

\section{Acknowledgments}

The work of D.H. is supported by the UK Science and Technology Facilities Council [grants ST/L002760/1 and ST/K004883/1].

\section{References}

[1] P. Bärnreuther, M. Czakon and A. Mitov, Phys. Rev. Lett. 109, 132001 (2012) [arXiv:1204.5201 [hep-ph]].

[2] M. Czakon and A. Mitov, JHEP 1212, 054 (2012) [arXiv:1207.0236 [hep-ph]].

[3] M. Czakon and A. Mitov, JHEP 1301, 080 (2013) [arXiv:1210.6832 [hep-ph]].

[4] M. Czakon, P. Fiedler and A. Mitov, Phys. Rev. Lett. 110, no. 25, 252004 (2013) [arXiv:1303.6254 [hep-ph]]. 
[5] M. Cacciari, M. Czakon, M. Mangano, A. Mitov and P. Nason, Phys. Lett. B 710, 612 (2014) [arXiv:1111.5869 [hep-ph]].

[6] M. Czakon and A. Mitov, Comput. Phys. Commun. 185, 2930 (2014) [arXiv:1112.5675 [hep-ph]].

[7] S. Chatrchyan et al. [CMS Collaboration], JHEP 1211, 067 (2012) [arXiv:1208.2671 [hep-ex]].

[8] S. Chatrchyan et al. [CMS Collaboration], JHEP 1402, 024 (2014) [JHEP 1402, 102 (2014)] [arXiv:1312.7582 [hep-ex], arXiv:1312.7582].

[9] G. Aad et al. [ATLAS Collaboration], Eur. Phys. J. C 74, no. 10, 3109 (2014) [arXiv:1406.5375 [hep-ex]].

[10] V. Khachatryan et al. [CMS Collaboration], arXiv:1510.05302 [hep-ex].

[11] [ATLAS Collaboration] ATLAS-CONF-2015-049.

[12] S. Chatrchyan et al. [CMS Collaboration], Eur. Phys. J. C 73, no. 3, 2339 (2013) [arXiv:1211.2220 [hep-ex]].

[13] G. Aad et al. [ATLAS Collaboration], Phys. Rev. D 90, no. 7, 072004 (2014) [arXiv:1407.0371 [hep-ex]].

[14] G. Aad et al. [ATLAS Collaboration], JHEP 1506, 100 (2015) [arXiv:1502.05923 [hep-ex]].

[15] V. Khachatryan et al. [CMS Collaboration], arXiv:1505.04480 [hep-ex].

[16] G. Aad et al. [ATLAS Collaboration], arXiv:1510.03818 [hep-ex].

[17] [CMS Collaboration] CMS-PAS-TOP-15-005.

[18] [CMS Collaboration] CMS-PAS-TOP-15-010.

[19] V. Ahrens, A. Ferroglia, M. Neubert, B. D. Pecjak and L. L. Yang, JHEP 1009, 097 (2010) [arXiv:1003.5827 [hep-ph]].

[20] V. Ahrens, A. Ferroglia, M. Neubert, B. D. Pecjak and L. L. Yang, JHEP 1109, 070 (2011) [arXiv:1103.0550 [hep-ph]].

[21] N. Kidonakis, Phys. Part. Nucl. 45, no. 4, 714 (2014) [arXiv:1210.7813 [hep-ph]].

[22] N. Kidonakis, Phys. Rev. D 91, no. 3, 031501 (2015) [arXiv:1411.2633 [hep-ph]].

[23] M. Guzzi, K. Lipka and S. O. Moch, JHEP 1501, 082 (2015) [arXiv:1406.0386 [hep-ph]].

[24] A. Broggio, A. S. Papanastasiou and A. Signer, JHEP 1410, 98 (2014) [arXiv:1407.2532 [hep-ph]].

[25] M. Czakon, D. Heymes and A. Mitov, arXiv:1511.00549 [hep-ph].

[26] S. Catani and M. H. Seymour, Nucl. Phys. B 485, 291 (1997) [Nucl. Phys. B 510, 503 (1998)] doi:10.1016/S0550-3213(96)00589-5 [hep-ph/9605323].

[27] S. Frixione, Z. Kunszt and A. Signer, Nucl. Phys. B 467, 399 (1996) doi:10.1016/0550-3213(96)00110-1 [hep-ph/9512328].

[28] G. Abelof, A. Gehrmann-De Ridder and I. Majer, arXiv:1506.04037 [hep-ph].

[29] G. Abelof and A. Gehrmann-De Ridder, JHEP 1412, 076 (2014) [arXiv:1409.3148 [hep-ph]].

[30] R. Bonciani, S. Catani, M. Grazzini, H. Sargsyan and A. Torre, arXiv:1508.03585 [hep-ph].

[31] M. Czakon, Phys. Lett. B 693, 259 (2010) [arXiv:1005.0274 [hep-ph]].

[32] M. Czakon, Nucl. Phys. B 849 (2011) 250 [arXiv:1101.0642 [hep-ph]]. 
[33] M. Czakon, P. Fiedler and A. Mitov, Phys. Rev. Lett. 115, no. 5, 052001 (2015) [arXiv:1411.3007 [hep-ph]].

[34] M. Czakon and D. Heymes, Nucl. Phys. B 890, 152 (2014) [arXiv:1408.2500 [hep-ph]].

[35] S. Catani and M. Grazzini, Nucl. Phys. B 570 (2000) 287 [hep-ph/9908523].

[36] J. M. Campbell and E. W. N. Glover, Nucl. Phys. B 527, 264 (1998) [hep-ph/9710255].

[37] S. Catani and M. Grazzini, Phys. Lett. B 446, 143 (1999) [hep-ph/9810389].

[38] V. Del Duca, A. Frizzo and F. Maltoni, Nucl. Phys. B 568, 211 (2000) [hep-ph/9909464].

[39] Z. Bern, L. J. Dixon, D. C. Dunbar and D. A. Kosower, Nucl. Phys. B 425, 217 (1994) [hep-ph/9403226].

[40] Z. Bern, V. Del Duca and C. R. Schmidt, Phys. Lett. B 445, 168 (1998) [hep-ph/9810409].

[41] D. A. Kosower, Nucl. Phys. B 552, 319 (1999) [hep-ph/9901201].

[42] D. A. Kosower and P. Uwer, Nucl. Phys. B 563, 477 (1999) [hep-ph/9903515].

[43] Z. Bern, V. Del Duca, W. B. Kilgore and C. R. Schmidt, Phys. Rev. D 60, 116001 (1999) [hep-ph/9903516].

[44] G. Somogyi and Z. Trocsanyi, JHEP 0701, 052 (2007) [hep-ph/0609043].

[45] S. Catani and M. Grazzini, Nucl. Phys. B 591, 435 (2000) [hep-ph/0007142].

[46] S. Catani, S. Dittmaier and Z. Trocsanyi, Phys. Lett. B 500, 149 (2001) [hep-ph/0011222].

[47] I. Bierenbaum, M. Czakon and A. Mitov, Nucl. Phys. B 856, 228 (2012) [arXiv:1107.4384 [hep-ph]].

[48] A. Ferroglia, M. Neubert, B. D. Pecjak and L. L. Yang, JHEP 0911, 062 (2009) [arXiv:0908.3676 [hep-ph]].

[49] M. Bury and A. van Hameren, Comput. Phys. Commun. 196 (2015) 592 [arXiv:1503.08612 [hep-ph]].

[50] J. G. Körner, Z. Merebashvili and M. Rogal, Phys. Rev. D 71, 054028 (2005) [hep-ph/0412088].

[51] C. Anastasiou and S. M. Aybat, Phys. Rev. D 78, 114006 (2008) [arXiv:0809.1355 [hep-ph]].

[52] B. Kniehl, Z. Merebashvili, J. G. Körner and M. Rogal, Phys. Rev. D 78, 094013 (2008) [arXiv:0809.3980 [hep-ph]].

[53] S. Dittmaier, P. Uwer and S. Weinzierl, Phys. Rev. Lett. 98, 262002 (2007) [hep-ph/0703120 [HEP-PH]].

[54] S. Dittmaier, P. Uwer and S. Weinzierl, Eur. Phys. J. C 59, 625 (2009) [arXiv:0810.0452 [hep-ph]].

[55] M. Czakon, Phys. Lett. B 664 (2008) 307 [arXiv:0803.1400 [hep-ph]].

[56] P. Bärnreuther, M. Czakon and P. Fiedler, JHEP 1402, 078 (2014) [arXiv:1312.6279 [hep-ph]].

[57] R. Bonciani, A. Ferroglia, T. Gehrmann, A. von Manteuffel and C. Studerus, JHEP 1312, 038 (2013) [arXiv:1309.4450 [hep-ph]].

[58] R. Bonciani, A. Ferroglia, T. Gehrmann, A. von Manteuffel and C. Studerus, JHEP 1101, 102 (2011) [arXiv:1011.6661 [hep-ph]].

[59] R. Bonciani, A. Ferroglia, T. Gehrmann and C. Studerus, JHEP 0908, 067 (2009) [arXiv:0906.3671 [hep-ph]]. 
[60] R. Bonciani, A. Ferroglia, T. Gehrmann, D. Maitre and C. Studerus, JHEP 0807, 129 (2008) [arXiv:0806.2301 [hep-ph]].

[61] A. D. Martin, W. J. Stirling, R. S. Thorne and G. Watt, Eur. Phys. J. C 63, 189 (2009) [arXiv:0901.0002 [hep-ph]].

[62] M. Cacciari, S. Frixione, M. L. Mangano, P. Nason and G. Ridolfi, JHEP 0809, 127 (2008) [arXiv:0804.2800 [hep-ph]].

[63] J. M. Campbell and R. K. Ellis, arXiv:1204.1513 [hep-ph].

[64] P. Nason, S. Dawson and R. K. Ellis, Nucl. Phys. B 303, 607 (1988); Nucl. Phys. B 327, 49 (1989) [Erratum-ibid. B 335, 260 (1990)].

[65] N. Kidonakis and G. F. Sterman, Nucl. Phys. B 505, 321 (1997) [hep-ph/9705234].

[66] E. Laenen, G. Oderda and G. F. Sterman, Phys. Lett. B 438, 173 (1998) [hep-ph/9806467].

[67] N. Kidonakis, E. Laenen, S. Moch and R. Vogt, Phys. Rev. D 64, 114001 (2001) [hep-ph/0105041].

[68] K. Hagiwara, Y. Sumino and H. Yokoya, Phys. Lett. B 666, 71 (2008) [arXiv:0804.1014 [hep-ph]].

[69] Y. Kiyo, J. H. Kuhn, S. Moch, M. Steinhauser and P. Uwer, Eur. Phys. J. C 60, 375 (2009) [arXiv:0812.0919 [hep-ph]].

[70] R. Frederix and F. Maltoni, JHEP 0901, 047 (2009) doi:10.1088/1126-6708/2009/01/047 [arXiv:0712.2355 [hep-ph]].

[71] CMS Collaboration [CMS Collaboration], CMS-PAS-TOP-15-011.

[72] G. Aad et al. [ATLAS Collaboration], arXiv:1511.04716 [hep-ex].

[73] A. Ferroglia, B. D. Pecjak and L. L. Yang, Phys. Rev. D 86, 034010 (2012) doi:10.1103/PhysRevD.86.034010 [arXiv:1205.3662 [hep-ph]].

[74] A. Ferroglia, S. Marzani, B. D. Pecjak and L. L. Yang, JHEP 1401, 028 (2014) doi:10.1007/JHEP01(2014)028 [arXiv:1310.3836 [hep-ph], arXiv:1310.3836].

[75] A. Ferroglia, B. D. Pecjak, D. J. Scott and L. L. Yang, arXiv:1512.02535 [hep-ph].

[76] M. Brucherseifer, F. Caola and K. Melnikov, JHEP 1304, 059 (2013) doi:10.1007/JHEP04(2013)059 [arXiv:1301.7133 [hep-ph]].

[77] J. Gao, C. S. Li and H. X. Zhu, Phys. Rev. Lett. 110, no. 4, 042001 (2013) doi:10.1103/PhysRevLett.110.042001 [arXiv:1210.2808 [hep-ph]]. 